\title{
HMMR Potential as a Diagnostic and Prognostic Biomarker of Cancer - Speculation Based on a Pan- cancer Analysis
}

\section{junyi Shang}

Henan Provincial People's Hospital https://orcid.org/0000-0002-1336-4941

Yong Qi ( $\sim$ qiyong@zzu.edu.cn )

People's Hospital of Zhengzhou University https://orcid.org/0000-0002-2653-1307

\section{Research}

Keywords: HMMR, Pan-cancer, Prognosis, Immune infiltration

Posted Date: November 30th, 2021

DOI: https://doi.org/10.21203/rs.3.rs-1091833/v1

License: (c) (i) This work is licensed under a Creative Commons Attribution 4.0 International License.

Read Full License 


\section{Abstract}

\section{Background}

Although the status of universal upregulation for the Hyaluronan-Mediated Motility Receptor (HMMR) in pan-cancer is still unknown, HMMR has been found to be upregulated and associated with poor prognosis for some tumors. The aims of our study included: exploring HMMR expression in different tumor types using The Cancer Genome Atlas (TCGA) or other public databases for a pan-cancer analysis, exploring the relationship between HMMR and tumor prognosis, and exploring the role of HMMR in tumor immunity.

Methods

We evaluated HMMR expression in pan-cancer and in different tumor cells using the TCGA, TIMER, ONCOMINE, and CCLE databases. Next, we analyzed the relationship between HMMR and the prognosis of different tumors subtypes. Receiver Operating Characteristic (ROC) curves were constructed based on the expression of HMMR, and analyzed using a correlation between HMMR and pathological stage and tumor immunity in pan-cancer. In the final step, we analyzed protein-protein interactions using STRING and implemented a GO/KEGG enrichment analysis.

Results

No matter the pairing or unpairing of data, HMMR expression generally increased as compared to corresponding normal tissue. Based on a CCLE study, our results indicated that HMMR is widely expressed in a variety of tumor cells. For most tumor types, high HMMR expression was associated with reduced Overall Survival (OS), Return to Functional Status (RFS), and Platinum Free Interval (PFI). ROC curves indicated that HMMR displays a high degree of prediction potential for most tumor types. In pancancer, HMMR is correlated with some clinical staging, immune cells, and immune checkpoints for some tumors. The results of the GO/KEGG enrichment analysis for proteins most closely related to HMMR, indicating that the most highly enriched pathways are all related to tumor development.

Conclusion

Our pan-cancer analysis of HMMR suggests that HMMR can be used as a potential diagnostic and prognostic indicator of pan-cancer and that HMMR may be involved in tumor development.

\section{Introduction}

Cancer occurrence caused by multiple biologic processes is a life-threatening disease and, worldwide, imposes a substantial economic burden (1-3)[1, 2]. Each year, \{Ferlay, 2019 \#2414\}tens of thousands of people die of cancer. Due to the lack of effective diagnosis and therapy, cancer causes a higher mortality rate in less developed countries. Early diagnosis and treatment can greatly reduce cancer morbidity, and effective treatments can increase patient survival rates. Treatments for cancer includes surgery, 
chemotherapy, radiation, targeted therapy, immunotherapy, etc. Accurate cancer diagnosis requires effective predictive markers. The implementation of immune therapy from immune-suppressor checkpoints, such as Programmed Cell Death Protein 1/Programmed Death-Ligand 1 (PD1/PDL1) expression, is often beneficial. Although radical surgery is currently the most effective treatment, many cancer patients do not benefit from surgery(4). Chemotherapy, radiotherapy, targeted therapies and immunotherapies are not applicable to all patients(5)[2]. As such, the discovery and application of new diagnostic markers and therapeutic targets is never out-of-date.

Exposing general phenomena related to cancer has become one of the major hotspots in cancer research. Bioinformatics have made it easier to conduct commonality research related to tumors. One such commonality is the role of the Hyaluronan-Mediated Motility Receptor (HMMR) in breast cancer(6,7), pancreatic cancer(8), and lung adenocarcinomas(9). To date, the role of HMMR in pan-cancer has not yet been explored. The purpose of this study was to analyze the expression of HMMR in pan-cancer and its potential value for diagnostic and prognostic use.

\section{Methods}

\section{The expression of MHHR in pan-cancer}

The GTEx dataset was obtained from TCGA (https://portal.gdc.cancer.gov/) and processed through Toil. RNAseq data were downloaded from the HTSeq-FPKM data of TCGA Pan-Cancer with retention from groups: (Adrenocortical carcinoma) ACC, (Bladder Urothelial Carcinoma) BLCA, (Breast invasive carcinoma) BRCA, (Cervical squamous cell carcinoma and endocervical adenocarcinoma) CESC, (Cholangiocarcinoma) CHOL, (Colon adenocarcinoma) COAD, (Lymphoid Neoplasm Diffuse Large B-cell Lymphoma) DLBC, (Esophageal carcinoma) ESCA, (Glioblastoma multiforme) GBM, (Head and Neck squamous cell carcinoma) HNSC, (Kidney Chromophobe) KICH, (Kidney renal clear cell carcinoma) KIRC, (Kidney renal papillary cell carcinoma) KIRP, (Acute Myeloid Leukemia) LAML, (Lower Grade GLioma) LGG, (Liver hepatocellular carcinoma) LIHC, (Lung adenocarcinoma) LUAD, (Lung squamous cell carcinoma) LUSC, (Mesothelioma) MESO, (Ovarian serous cystadenocarcinoma) OV, (Pancreatic adenocarcinoma) PAAD, (Pheochromocytoma and Paraganglioma) PCPG, (Prostate adenocarcinoma) PRAD, (Rectum adenocarcinoma) READ, (Sarcoma) SARC, (Skin Cutaneous Melanoma) SKCM, (Stomach adenocarcinoma) STAD, (Testicular Germ Cell Tumor) TGCT, (Thyroid carcinoma) THCA, (Thymoma) THYM, (Uterine Corpus Endometrial Carcinoma) UCEC, (Uterine Carcinosarcoma) UCS, and (Uveal Melanoma) UVM. The RNAseq data of tumors and paracancerous tissues in different cancers also came from the HTSeq-FPKM data of TCGA Pan-Cancer for retention paired samples. The differential expressions of HMMR in GTEx, in paired or unpaired samples from TCGA, were all statistically processed using $\mathrm{R}$ (Version 3.6.3), and ggplot2 [Version 3.3.3] for visualization. For analyzing differential HMMR expression between tumor and adjacent normal tissue, we also used TIMER (https://cistrome.shinyapps.io/timer/)(10). To validate the reliability of TCGA, we used ONCOMINE (https://www.oncomine.org), a public database for retrieving HMMR expression in different cancers. Finally, the HMMR expression matrix for 946 cell lines from 22 types of cancers were obtained from the 
CCLE (https://portals.broadinstitute.org/ccle/about). To construct HMMR distributions in different tumoral tissues, we used R (v4.0.3) and ggplot2 (v3.3.3).

\section{The relationship between HMMR and Overall Survival (OS)}

We used the Kaplan-Meier Plotter (https://www.kmplot.com) to analyze the association between HMMR and OS in pan-cancer. Next, prognostic data for 33 types of cancer were downloaded from TCGA. Samples were then distributed into low and high groups based on HMMR expression. The R survivminer package (Version 0.4.9) was used for visualization, and the survival package (Version 3.2-10) was used

for statistical analyses of the survival data. The HR and $P$ values for each tumor prognosis based HMMRexpression were determined using the Cox regression model, then constructed the forest plots.

\section{The relationship between HMMR and Recurrence Free Survival (RFS) and Progress Free Interval (PFI)}

The Kaplan Meier plotter was also used for analyzing the relationship between HMMR and RFS in pancancer. We downloaded pan-cancer clinical data from TCGA, divided the data into a high HMMR expression group and a low HMMR expression group, and then obtained the HR and P for each type of cancer based on HMMR expression using a Cox regression model. Statistical analyses and visualization were determined using the $\mathrm{R}$ survminer package (Version 0.4.9) and the survival package (Version 3.210). We employed forest plots for displaying the PFI for each tumor type.

\section{ROC curves based on HMMR expression}

RNAseq data were obtained from the TCGA dataset. ROC curves were constructed for predicting normal cells or cancer. We used R (Version 3.6.3) for statistical analyses and visualization. The pROC package (Version 1.17.0.1) and the ggplot2 package (Version 3.3.3) were also simultaneously used for analyses and visualization, and for the calculation of the confidence intervals and curve areas.

\section{The correlation between HMMR and clinical stage}

RNAseq and clinical information were obtained from TCGA. Normal and control samples were removed. We retained samples using clinical information. An analysis was implemented for HMMR and the Tstage. A Kruskal-Wallis test was employed for statistical analyses. For statistical analyses and visualization, we utilized R (Version 3.6.3). The program ggplot2 (Version 3.3.3) was also used for visualization.

\section{The relationship between HMMR and immune infiltration}

The RNA-seq data of 33 tumors types was downloaded from the TCGA database. mRNA expression data was also downloaded from 33 tumors with paired normal tissue samples. Immune-related assessment was performed using the immunedeconv package. The package consists of six integrated algorithms, including TIMER, xCell(11), MCP-counter(12), CIBERSORT(13), EPIC(14), and quanTIseq(15). R (v4.0.3) 
was used for statistical analyses. The significance of two groups was performed using a Wilcoxon rank sum test.

\section{The correlation between HMMR and immune checkpoints}

SIGLEC15, ID01, CD274, HAVCR2, PDCD1, CTLA4, LAG3, and PDCD1LG2 are immune checkpoint-related transcripts. We extracted these eight gene-expressions of 33 tumor types from TCGA and calculated correlations between HMMR and checkpoint-related transcripts. We used R (Version 4.0.3) for statistical analyses. A rank sum test was used to detect two sets of data. P-values $\leq 0.05$ were considered statistically significant.

\section{The correlation between HMMR and Tumor Mutation Burden (TMB) and MicroSatellite Instability (MSI)}

The RNA-seq database of 33 tumor patients was downloaded from TCGA, and each tumor had matched mRNA expression data. TMB was derived from the article by Thorsson et al(16). MSI was derived from the article by Bonneville et al(17). Statistical analyses were performed using R software (Version 4.0.3). A rank sum test was employed on two sets of data. $P$ value $<0.05$ was considered statistically significant.

\section{Analysis of molecular correlates}

The STRING Database (https://string-db.org/) is a Protein-Protein Interaction (PPI) analysis database that can predict protein-protein interactions of known proteins. HMMR was entered into the STRING website by selecting Homo sapiens. The top ten HMMR-related proteins were then obtained and the results were downloaded. For constructing the PPI network, R (Version 3.6.3) and the igraph package (Version 1.2.6) were employed for statistical analyses and visualization. In the same manner, we obtained the top 50 proteins most tightly associated with HMMR. An enrichment analysis was performed for GO and KEGG terms. The ggplot2 package (Version 3.3.3) and the clusterProfile package (Version 3.14.3) were used for statistical analysis and visualization.

\section{Results}

\section{Differential HMMR expression in various cancers}

HMMR mRNA expression levels in (Adrenocortical carcinoma) ACC, (Bladder Urothelial Carcinoma) BLCA, (Breast invasive carcinoma) BRCA, (Cervical squamous cell carcinoma and endocervical adenocarcinoma) CESC, (Cholangiocarcinoma) CHOL, (Colon adenocarcinoma) COAD, (Lymphoid Neoplasm Diffuse Large B-cell Lymphoma) DLBC, (Esophageal carcinoma) ESCA, (Glioblastoma multiforme) GBM, (Head and Neck squamous cell carcinoma) HNSC, (Kidney Chromophobe) KICH, (Kidney renal clear cell carcinoma) KIRC, (Kidney renal papillary cell carcinoma) KIRP, (Lower Grade GLioma) LGG, (Liver hepatocellular carcinoma) LIHC, (Lung adenocarcinoma) LUAD, (Lung squamous cell carcinoma) LUSC, (Ovarian serous cystadenocarcinoma) OV, (Pancreatic adenocarcinoma) PAAD, (Pheochromocytoma and Paraganglioma) PCPG, (Prostate adenocarcinoma) PRAD, (Rectum adenocarcinoma) READ, (Skin Cutaneous Melanoma) SKCM, (Stomach adenocarcinoma) STAD, (Thyroid 
carcinoma) THCA, (Thymoma) THYM, (Uterine Corpus Endometrial Carcinoma) UCEC, and (Uterine Carcinosarcoma) UCS were determined to be higher than in normal tissues, with the exception of (Acute Myeloid Leukemia) LAML and (Testicular Germ Cell Tumor) TGCT (Figure 1A). A Wilcoxon rank sum unpaired test for TCGA data indicated that the expression of HMMR mRNA in BLCA, BRCA, CESC, CHOL, COAD, ESCA, GBM, HNSC, KIRC, KIRP, LIHC, LUAD, LUSC, PRAD, READ, STAD, THCA, and UCEC was higher than in normal tissue (Figure 1B). For TCGA paired samples, HMMR mRNA expression in BLCA, BRCA, $\mathrm{CHOL}$, ESCA, HNSC, KICH, KIRC, KIRP, LIHC, LUAD, LUSC, PRAD, READ, STAD, and UCEC were all at higher levels in tumor tissues than in matched normal adjacent tissues (Figure 1C).

The expression of HMMR in pan-cancer was also analyzed using the TIMER database. HMMR expression was at a higher level in BLCA, BRCA, CESC, CHOL, COAD, ESCA, GBM, HNSC, KIRC, KIRP, LIHC, LUAD, LUSC, PRAD, READ, SKCM, STAD, THCA, and UCEC as compared to normal tissue (Figure 1D). We also verified differential HMMR expression between tumor and normal tissue using the ONCOMINE software. With the exception of leukemia and some other cancers, HMMR expression in tumor tissues was significantly higher than in normal tissues (Figure 1E). Finally, we used the Cancer Cell Line Encyclopedia (CCLE) to profile HMMR expression across various types of cancer (Figure 1F).

Figure 1. HMMR expression in different types of tumors. The abscissa represents samples in different groups and the ordinate shows the distribution of HMMR expression. $n s, p \geq 0.05 ;{ }^{*}, p<0.05 ; * \star, p<0.01$; $\star \star \star, p<0.001$. (A) HMMR expression analysis in pan-cancer for GTEx datasets. (B) Differential HMMR expression in tumor tissues as compared to normal tissues. (C) Different HMMR expression between tumor and paired adjacent normal tissues utilized from the TCGA dataset. (D) HMMR expression for the TIMER dataset. (E) HMMR expression in different tissues for the ONCOMINE dataset, blue represents lower expression and red represents higher expression. (F) HMMR expression in different tumor types using the CCLE database.

\section{The relationship between HMMR expression and Overall Survival (OS)}

Figure 2A-L represents the relationship between HMMR expression and OS across different tumor types. For bladder carcinoma, breast cancer, esophageal adenocarcinoma, head-neck squamous cell carcinoma, kidney renal clear cell carcinoma, kidney renal papillary cell carcinoma, liver hepatocellular carcinoma, lung adenocarcinoma, pancreatic ductal adenocarcinoma, sarcoma, and stomach adenocarcinoma high HMMR expression indicated reduced OS. For thymoma and uterine corpus endometrial carcinoma, high HMMR expression predicted better OS.

Figure 2. The relationship between HMMR gene expression and OS: (A) bladder carcinoma, (B) breast cancer, (C) esophageal adenocarcinoma, (D) head-neck squamous cell carcinoma, (E) kidney renal clear cell carcinoma, (F) kidney renal papillary cell carcinoma, (G) liver hepatocellular carcinoma, (H) lung adenocarcinoma, (I) pancreatic ductal adenocarcinoma, (J) sarcoma, (K) stomach adenocarcinoma, (M) thymoma, $(\mathrm{N})$ uterine corpus endometrial carcinoma, and $(\mathrm{L})$ the forest plot of HRs for OSs in different tumor types. 


\section{The relationship of HMMR to RFS and PFI}

For bladder carcinoma, breast cancer, esophageal adenocarcinoma, kidney renal clear cell carcinoma, kidney renal papillary cell carcinoma, liver hepatocellular carcinoma, lung adenocarcinoma, pancreatic ductal adenocarcinoma, sarcoma, thyroid carcinoma and uterine corpus endometrial carcinoma, patients with higher HMMR expression were predicted to have a reduced RFS (Figure 3A-K). In Figure 3L, the correlation between HMMR and PFI for the various tumor types are displayed using forest plots.

Figure 3. A RFS survival analysis of HMMR expression in pan-cancer: (A) bladder carcinoma, (B) breast cancer, (C) esophageal adenocarcinoma, (D) kidney renal clear cell carcinoma, (E) kidney renal papillary cell carcinoma, $(F)$ liver hepatocellular carcinoma, $(G)$ lung adenocarcinoma, $(H)$ pancreatic ductal adenocarcinoma, $(\mathrm{I})$ sarcoma, $(\mathrm{J})$ thyroid carcinoma, $(\mathrm{K})$ uterine corpus endometrial carcinoma, and $(\mathrm{L})$ PFI forest plots for HMMR expression in pan-cancer.

\section{ROC curves constructed by HMMR expression}

For LAML, BRCA, CESC, CHOL, ESCA, GBM, (Glioma) GBMLGG, HNSC, LGG, LIHC, LUAD, LUSC, OV, PAAD, STAD, UCEC, and UCS, HMMR has a high accuracy in predicting tumor and normal outcomes. For BLCA, COAD, (Colon adenocarcinoma/Rectum adenocarcinoma Esophageal carcinoma) COADREAD, KIRC, PRAD, READ, and TGCT, HMMR also has a certain accuracy in predicting tumor and normal outcomes(Figure 4).

Figure 4. The ROC curve of HMMR in predicting tumor and normal outcomes. For Areas Under the ROC Curve (AUC) (which are between 0.5 and 1 ) the closer the value is to 1, the better the diagnostic value. HMMR displays poor accuracy for AUCs between 0.5 and 0.7. HMMR displays certain accuracy when AUC is between 0.7 and 0.9 , and good accuracy when AUC is above 0.9 .

\section{The correlation between HMMR expression and tumor stage}

Based on a correlation analysis between HMMR and different tumor stages, ACC, BRCA, KIRC, KIRP, LUAD, LIHC, LUADLUSC, LUSC, TGCT, and HMMR had certain predictive accuracy for the T stage(Figure 5A-I).

Figure 5. A statistical correlation analysis between HMMR and the pathological T stage. The horizontal axis represents different $T$ stage, the vertical axis represents HMMR expression. $n s, p \geq 0.05 ;{ }^{*}, p<0.05$; $\star \star, p<0.01 ; * \star *, p<0.001$.

\section{The correlation analysis between HMMR and immune cells}

Our research separately used CIBERSORT (Figure 6A), xCell (Figure 6B), EPIC (Figure 6C), TIMER (Figure 6D), quanTIseq (Figure 6E), and MCP-counter (Figure 6F) to calculate the correlation between HMMR and immune cells in various tumor types. Figure 6 indicates that HMMR has correlations with multiple different types of immune cells for 33 tumor types $(P<0.05)$. 
Figure 6. The correlation between HMMR and different immune cells in various tumor types. The horizontal axis represents different tumor types, the vertical axis represents different immune scores, different colors represent the correlation coefficient, negative values indicate an inverse correlation, and positive values indicate a positive correlation. The stronger the correlation, the darker the color. Asterisks represent the degree of importance $\left({ }^{*} p\right) .{ }^{*}, p<0.05 ; * \star, p<0.01$; $* \star *, p<0.001$. (A) CIBERSORT, (B) $x$ Cell, (C) EPIC, (D) TIMER, (E) quanTIseq, and (F) MCP-counter.

\section{Heat maps for the relationship between HMMR expression and immune checkpoint-related genes for various tumor types}

SIGLEC15, ID01, CD274, HAVCR2, PDCD1, CTLA4, LAG3, and PDCD1LG2 are immune checkpoint-related genes. Figure 7 provides a heatmap of the correlation between immune checkpoints and HMMR in 33 tumor types from TCGA. In addition to PCPG, MESO, ESCA, and CHOL, HMMR was associated with multiple immune checkpoint-related genes $(P<0.05)$ (Figure 7$)$.

Figure 7. A heat map for the correlation between HMMR and immune checkpoint related genes in 33 tumor types from TCGA. The horizontal axis provides various immune checkpoint-related genes, and the vertical axis provides various tumor types. Each single box in the figure indicates the correlation between HMMR expression and the expression of immune checkpoint related genes in each tumor. Different colors represent different correlation coefficients. The stronger the color, the stronger the correlation. Asterisks represent the level of importance $\left({ }^{*} p\right), *, p<0.05 ; * \star, p<0.01$, and ${ }^{* *}, p<0.001$.

\section{The correlation of HMMR with TMB and SMI}

Figure 8 provides a Pearson correlation analyses for HMMR expression correlated with TMB (Figure 8A) and MSI (Figure 8B). The abscissa provides the correlation coefficient between the gene and TMB or MSI. The ordinate provides various tumors. Colors represent various $P$ values. The smaller the $p$ value, the bluer the color.

Figure 8. A correlation analysis for HMMR with TMB (A) and MSI (B) for different tumor types.

\section{Molecular correlation analyses}

We performed a STRING database pathway analysis. The strongest associated proteins to HMMR were LYVE1, TPX2, STAB2, ASPM, AURKA, BRCA1, BUB1, CD44, CDK1, and DLGAP5. The results are provided as a Protein-Protein Interaction (PPI) network (Figure 9A). We then performed a gene GO/KEGG analysis for the top 50 protein associations to HMMR (Figure 9B). Nuclear division and regulation of the cell cycle phase transition were the most enriched GO molecular function term. The most enriched KEGG pathway was the Cell cycle.

Figure 9. (A) The PPI network for the top 10 proteins associated with HMMR. (B) A GO/KEGG enrichment analysis for the top 50 proteins associated with HMMR. 


\section{Discussion}

HMMR is a type of hyaluronic acid receptor associated with cell movement(18)\{Antón, 2008 \#2489\}. HMMR influences brain development through regulating the spindle(19). Elevated HMMR expression is always associated with poor prognosis in breast cancer $(20,21)$. Some bioinformatics analysis findings have indicated that HMMR is the hub gene for some tumors $(22,23)$. However, the role of HMMR in all cancer types is largely unknown. The purpose of our study was to explore HMMR in pan-cancer.

HMMR is expressed at low levels in most healthy tissues. Our study of HMMR expression in pan-cancer found that HMMR was upregulated in most tumor types as compared to normal tissues. Our results indicated that HMMR maybe a marker of poor prognosis for some tumors. In order to verify this hypothesis, we separately repeated the analysis using TCGA, TIMER, and ONCOMINE, and found outcomes to be similar.

Tumor tissues are composed of multiple and complex cell types. Currently, the Cancer Cell Line Encyclopedia (CCLE) is the largest tumor cells database(24). As such, a thorough analysis of various tumor cells using the database is capable of reflecting the heterogeneity of tumor cells. Our analysis using the CCLE cell line database was, therefore, helpful for interpreting HMMR expression for various tumor cells. HMMR was found to be an independent prognostic indicator for prostate cancer(25). Based on our prognosis analysis, high HMMR expression predicts inferior survival for bladder carcinoma, breast cancer, esophageal adenocarcinoma, head-neck squamous cell carcinoma, kidney renal clear cell carcinoma, kidney renal papillary cell carcinoma, liver hepatocellular carcinoma, lung adenocarcinoma, pancreatic ductal adenocarcinoma, sarcoma, and stomach adenocarcinoma. Likewise, based on our pancancer analysis, for a variety of tumor types, a high level of HMMR is associated with inferior RFS and PFI. ROC curves revealed the predictive power of HMMR regarding tumors. When the area under the ROC curve is close to 1 , the predictive power of HMMR is stronger. With the TCGA database analysis, we found that the area under ROC curves were all above 0.9 for most tumor types, indicating that HMMR could be a potential diagnostic marker for these tumors. Past researchers have outlined HMMR levels that are upregulated in breast cancer and accompanied by poor pathologic stages and tumor size(26). In our pancancer analysis of tumor stage, we determined associations of HMMR with some tumor stages.

Immunotherapy is currently a very important hot treatment for cancer therapy and has become the firstline therapy for cancer patients. The essence of immunotherapy is the mobilization of immune cells to kill tumor cells. Changes within the tumor microenvironment inhibits the function of immune cells and stops immune cells from engulfing tumor cells, thereby, promoting tumor progression and migration. The immune escape of tumors can also suppress or block immune response. Immunotherapy began in 1983(27). Targeting immune checkpoint inhibitors is the most common type of immunotherapy, and PD1 and PDL1 are the most commonly used immune checkpoints(28). In recent years, additional potential immune checkpoints have also been determined $(29,30)$. The immune checkpoints expressed on immune cells belong to a class of immunosuppressive molecules that can regulate the activation of immunity. Immune checkpoint molecules cause the immune system to remain within a normal range, thus, the 
immune system does not become over-activated. Our research analyses included the correlation between HMMR expression and different types of immune cells. Our results indicate that HMMR could potentially become a new therapeutic target for tumors. With the exception of PD1 and PDL1, Tumor Mutational Burden (TMB) (31) and MicroSatellite Instability (MSI) (32) are also commonly used as predictive markers for immunotherapy. We also performed a correlation analysis between HMMR and MSI/MSI.

Studies of molecular interaction are favorable for analyzing molecular mechanisms. The purpose of the PPI network analysis was to explore protein-protein interactions. The STRING database provides a tool for analyzing the interaction between known and predicted proteins. Using a PPI analysis, we obtained the top 10 and top 50 proteins most closely related to HMMR. As seen in Figure 9B, the largest cluster pathways display the regulation of cell cycle phase transitions, nuclear division, cell cycle checkpoints, cell cycle, spindle, condensed chromosomes, and spindle pole. All of these pathways are closely related to the occurrence and development of cancer(33-35).

The shortcoming of our study is that pan-cancer analyses are difficult to simultaneously obtain for many types of tumor specimens. As such, the results could not be verified with our own clinical samples.

\section{Declarations}

\section{Our manuscript including all of the subheadings :}

Ethics approval and consent to participate: All of the data used in this study were downloaded directly from public databases, so ethical approval was not required for this study.

Consent for publication: Not applicable for this section.

Availability of data and materials: All data generated or analysed during this study are included in this published article.

Competing interests: The author(s) declare(s) that they have no competing interests.

Funding: Not applicable for this section.

Authors' contributions: (I) Conception and design: Yong Qi, Junyi Shang; (II) Administrative support: Yong Qi; (III) Provision of study materials or patients: Junyi Shang; (IV) Collection and assembly of data: Yong Qi, Junyi Shang; (V) Data analysis and interpretation: Junyi Shang; (VI) Manuscript writing: All authors; (VII) Final approval of manuscript: All authors.

Acknowledgements: Not applicable for this section.

\section{References}

1. Hanahan D, Weinberg RA. Hallmarks of cancer: the next generation. Cell. 2011 Mar 
4;144(5):646-74.

2 Torre LA, Siegel RL, Ward EM, et al. Global Cancer Incidence and Mortality Rates and Trends--An Update. Cancer 2Epidemiol Biomarkers Prev. 2016 Jan;25(1):16-27.

3 Ferlay J, Colombet M, Soerjomataram I, et al. Estimating the global cancer incidence and mortality in 2018: GLOBOCAN sources and methods. Int J Cancer. 2019 Apr 15;144(8):1941-1953.

4 Barbot AC, Mussault P, Ingrand P, et al. Assessing 2-month clinical prognosis in hospitalized patients with advanced solid tumors. J Clin Oncol. 2008 May 20;26(15):2538-43.

5 Zhu AX, Finn RS, Edeline J, et al. Pembrolizumab in patients with advanced hepatocellular carcinoma previously treated with sorafenib (KEYNOTE-224): a non-randomised, open-label phase 2 trial. Lancet Oncol. 2018 Jul;19(7):940-952.

Schwertfeger KL, Cowman MK, Telmer PG, et al. Hyaluronan, Inflammation, and Breast Cancer

Progression. Front Immunol. 2015 Jun 8;6:236.

Isakova JT, Vinnikov D, Kipen VN, et al. Gene-to-gene interactions and the association of

TP53, XRCC1, TNFa, HMMR, MDM2 and PALB2 with breast cancer in Kyrgyz females. Breast Cancer. 2020 Sep;27(5):938-946.

Lin A, Feng J, Chen X, et al. High levels of truncated RHAMM cooperate with dysfunctional p53 to accelerate the progression of pancreatic cancer. Cancer Lett. 2021 Aug 28;514:79-89.

Stevens LE, Cheung WKC, Adua SJ, et al. Extracellular Matrix Receptor Expression in

Subtypes of Lung Adenocarcinoma Potentiates Outgrowth of Micrometastases. Cancer Res. 2017 Apr 15;77(8):1905-1917.

Li B, Severson E, Pignon JC, et al. Comprehensive analyses of tumor immunity: implications for cancer immunotherapy. Genome Biol. 2016 Aug 22;17(1):174.

Aran D, Hu Z, Butte AJ. xCell: digitally portraying the tissue cellular heterogeneity landscape.

Genome Biol. 2017 Nov 15;18(1):220. doi: 10.1186/s13059-017-1349-1.

Becht E, Giraldo NA, Lacroix L, et al. Estimating the population abundance of tissueinfiltrating immune and stromal cell populations using gene expression. Genome Biol. 2016 Oct 20;17(1):218. 
Newman AM, Liu CL, Green MR, et al. Robust enumeration of cell subsets from tissue expression profiles. Nat Methods. 2015 May;12(5):453-7.

Racle J, de Jonge K, Baumgaertner P, et al. Simultaneous enumeration of cancer and immune cell types from bulk tumor gene expression data. Elife. 2017 Nov 13;6:e26476. Finotello F, Mayer C, Plattner C, et al. Molecular and pharmacological modulators of the tumor immune contexture revealed by deconvolution of RNA-seq data. Genome Med. 2019 May 24;11(1):34.

Thorsson V, Gibbs DL, Brown SD, et al. The Immune Landscape of Cancer [published correction appears in Immunity. 2019 Aug 20;51(2):411-412]. Immunity. 2018;48(4):812-830.e14.

Bonneville R, Krook MA, Kautto EA, et al. Landscape of Microsatellite Instability Across 39 Cancer Types. JCO Precis Oncol. 2017;2017:PO.17.00073.

18 Hardwick C, Hoare K, Owens R, et al. Molecular cloning of a novel hyaluronan receptor that mediates tumor cell motility. J Cell Biol. 1992 Jun;117(6):1343-50.

19 Li H, Kroll T, Moll J, et al. Spindle Misorientation of Cerebral and Cerebellar Progenitors Is a Mechanistic Cause of Megalencephaly. Stem Cell Reports. 2017 Oct 10;9(4):1071-1080.

20 Liu W, Ma J, Cheng Y, et al. HMMR antisense RNA 1, a novel long noncoding RNA, regulates the progression of basal-like breast cancer cells. Breast Cancer (Dove Med Press). 2016 Nov 24;8:223229.

21 Maxwell CA, Benítez J, Gómez-Baldó L, et al. Interplay between BRCA1 and RHAMM regulates epithelial apicobasal polarization and may influence risk of breast cancer. PLoS Biol. 2011 Nov;9(11):e1001199.

22 Lu D, Bai X, Zou Q, et al. Identification of the association between HMMR expression and progression of hepatocellular carcinoma via construction of a co-expression network. Oncol Lett. 2020 Sep;20(3):2645-2654.

23 Zhou Z, Li Y, Hao H, et al. Screening Hub Genes as Prognostic Biomarkers of Hepatocellular Carcinoma by Bioinformatics Analysis. Cell Transplant. 2019 Dec;28(1_suppl):76S-86S. 
24 Ghandi M, Huang FW, Jané-Valbuena J,et al. Next-generation characterization of the Cancer Cell Line Encyclopedia. Nature. 2019 May;569(7757):503-508.

25 Rizzardi AE, Rosener NK, Koopmeiners JS, et al. Evaluation of protein biomarkers of prostate cancer aggressiveness. BMC Cancer. 2014 Apr 5;14:244.

26 Yeh MH, Tzeng YJ, Fu TY,et al. Extracellular Matrix-receptor Interaction Signaling Genes Associated with Inferior Breast Cancer Survival. Anticancer Res. 2018 Aug;38(8):4593-4605. 27 Coley WB. The treatment of malignant tumors by repeated inoculations of erysipelas. With a report of ten original cases. 1893. Clin Orthop Relat Res. 1991 Jan;(262):3-11.

28 Amarnath S, Mangus CW, Wang JC, et al. The PDL1-PD1 axis converts human TH1 cells into regulatory T cells. Sci Transl Med. 2011 Nov 30;3(111):111ra120.

29 Fourcade J, Sun Z, Pagliano O, et al. CD8(+) T cells specific for tumor antigens can be rendered dysfunctional by the tumor microenvironment through upregulation of the inhibitory receptors BTLA and PD-1. Cancer Res. 2012 Feb 15;72(4):887-96.

30 Le Mercier I, Chen W, Lines JL, et al. VISTA Regulates the Development of Protective Antitumor Immunity. Cancer Res. 2014 Apr 1;74(7):1933-44.

31 Chalmers ZR, Connelly CF, Fabrizio D, et al. Analysis of 100,000 human cancer genomes reveals the landscape of tumor mutational burden. Genome Med. 2017 Apr 19;9(1):34.

32 Le DT, Durham JN, Smith KN, et al. Mismatch repair deficiency predicts response of solid tumors to PD-1 blockade. Science. 2017 Jul 28;357(6349):409-413.

33 Shihabudeen Haider Ali MS, Cheng X, Moran M, et al. LncRNA Meg3 protects endothelial function by regulating the DNA damage response. Nucleic Acids Res. 2019 Feb 20;47(3):1505-1522.

34 Dominguez D, Tsai YH, Gomez N, et al. A high-resolution transcriptome map of cell cycle reveals novel connections between periodic genes and cancer. Cell Res. 2016 Aug;26(8):946-62. 35 Bazzoun D, Adissu HA, Wang L, et al. Connexin 43 maintains tissue polarity and regulates mitotic spindle orientation in the breast epithelium. J Cell Sci. 2019 May 16;132(10):jcs223313. 
Figures

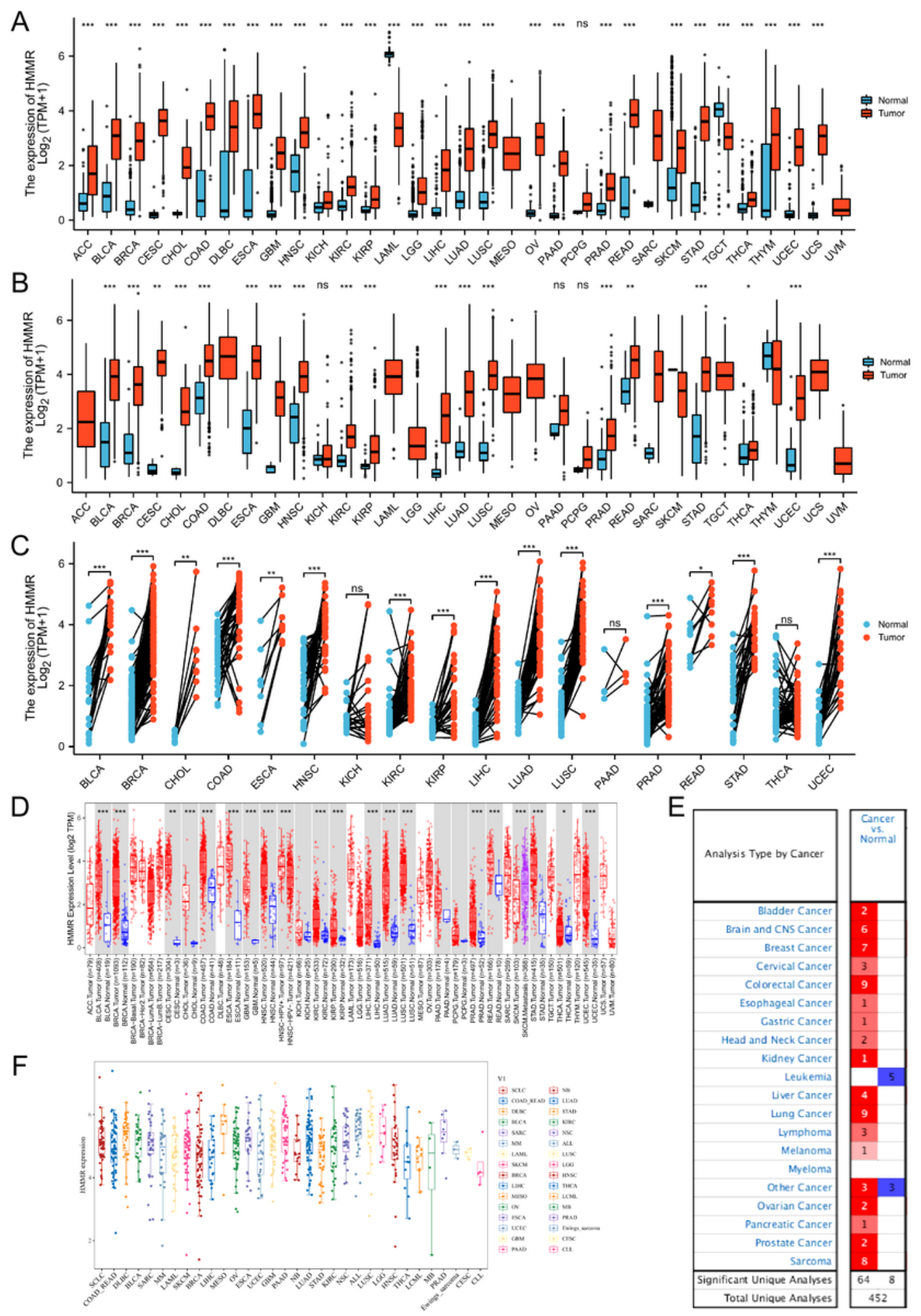

Figure 1 
HMMR expression in different types of tumors. The abscissa represents samples in different groups and the ordinate shows the distribution of HMMR expression. ns, $p \geq 0.05$; ${ }^{*}, p<0.05$; ${ }^{\star *}, p<0.01$; $* \star *, p<$ 0.001. (A) HMMR expression analysis in pan-cancer for GTEx datasets. (B) Differential HMMR expression in tumor tissues as compared to normal tissues. (C) Different HMMR expression between tumor and paired adjacent normal tissues utilized from the TCGA dataset. (D) HMMR expression for the TIMER dataset. (E) HMMR expression in different tissues for the ONCOMINE dataset, blue represents lower expression and red represents higher expression. (F) HMMR expression in different tumor types using the CCLE database. 

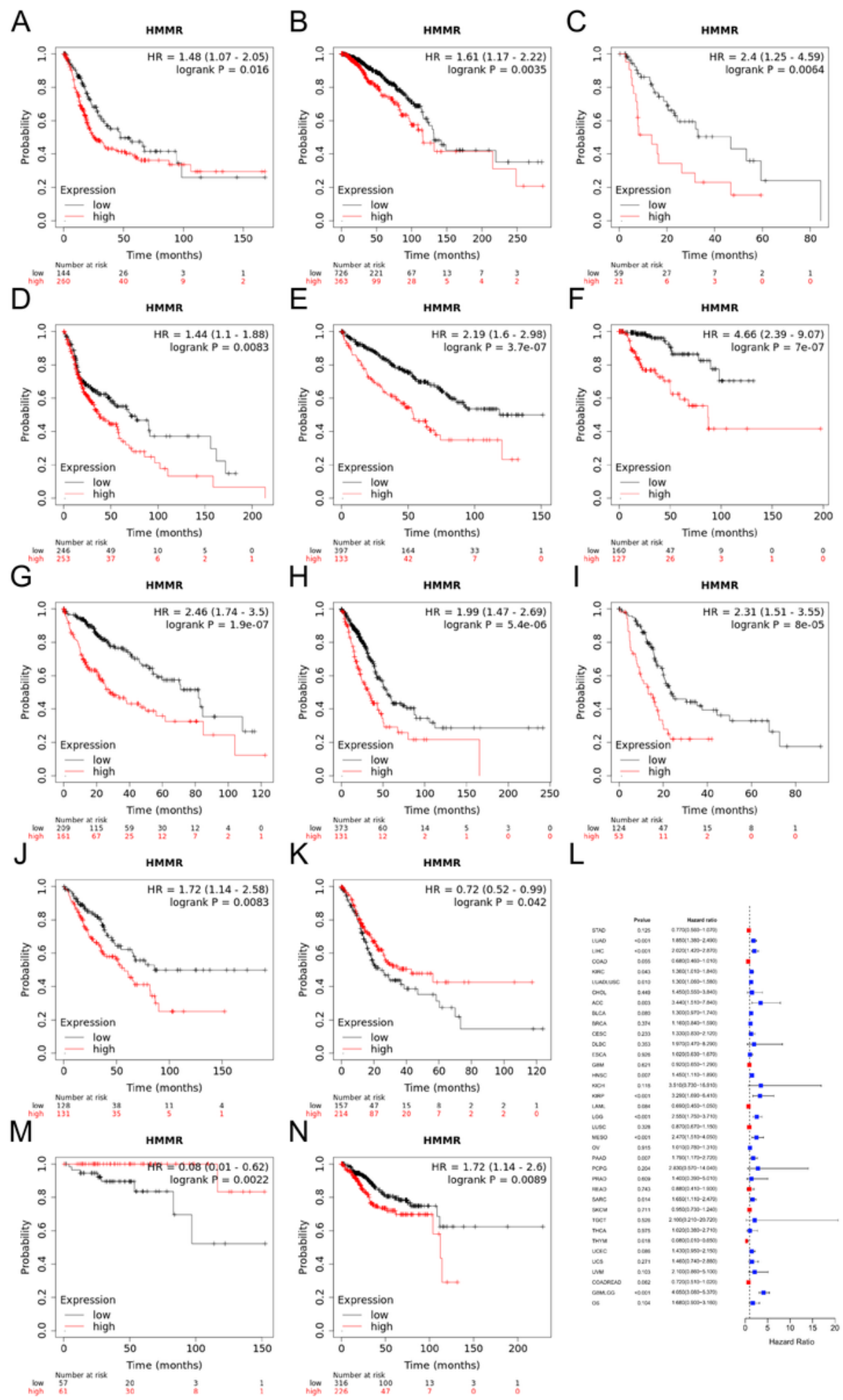

\section{Figure 2}

The relationship between HMMR gene expression and OS: (A) bladder carcinoma, (B) breast cancer, (C) esophageal adenocarcinoma, (D) head-neck squamous cell carcinoma, $(E)$ kidney renal clear cell carcinoma, (F) kidney renal papillary cell carcinoma, (G) liver hepatocellular carcinoma, (H) lung adenocarcinoma, (I) pancreatic ductal adenocarcinoma, (J) sarcoma, (K) stomach adenocarcinoma, (M) 
thymoma, $(\mathrm{N})$ uterine corpus endometrial carcinoma, and $(\mathrm{L})$ the forest plot of HRs for OSs in different tumor types.
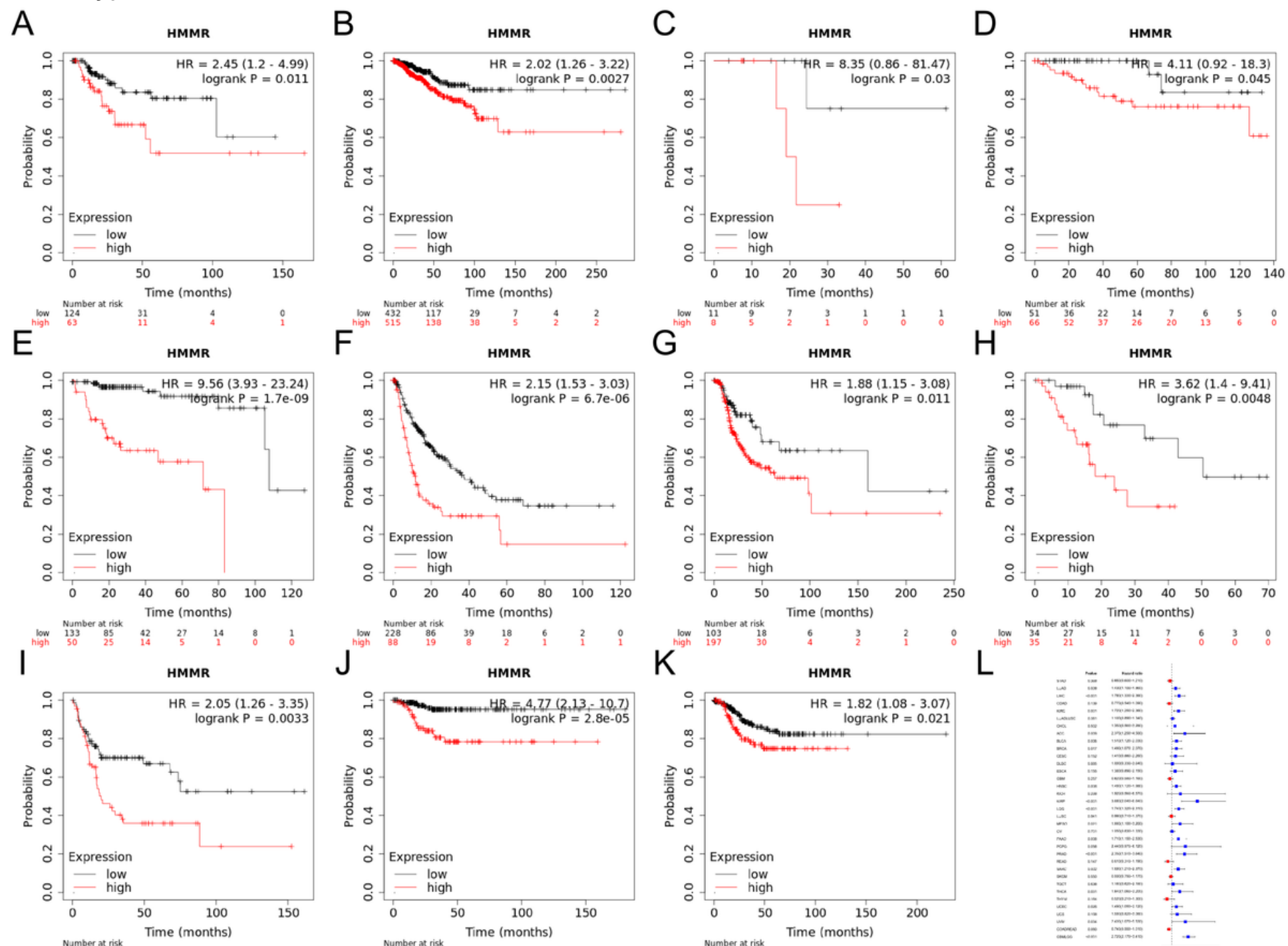

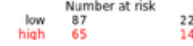

how $x_{25}$
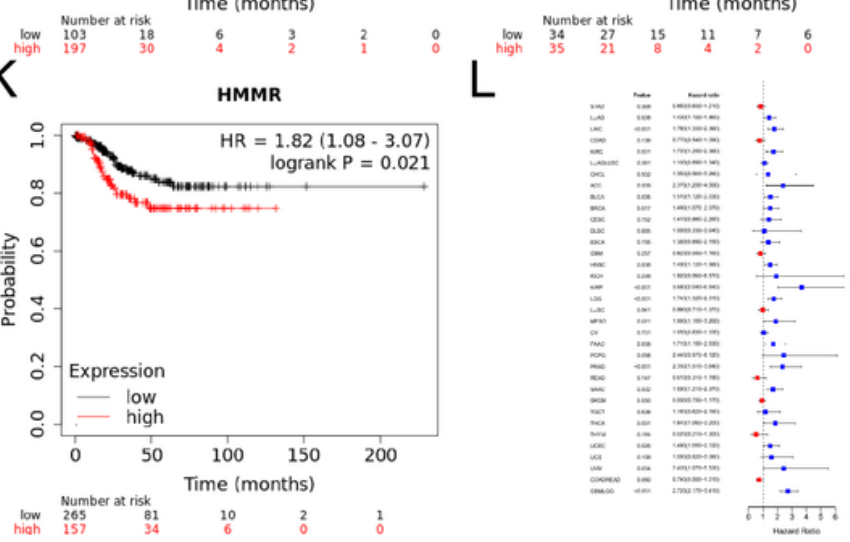

\section{Figure 3}

A RFS survival analysis of HMMR expression in pan-cancer: (A) bladder carcinoma, (B) breast cancer, (C) esophageal adenocarcinoma, (D) kidney renal clear cell carcinoma, $(E)$ kidney renal papillary cell carcinoma, (F) liver hepatocellular carcinoma, (G) lung adenocarcinoma, $(H)$ pancreatic ductal adenocarcinoma, (I) sarcoma, $(\mathrm{J})$ thyroid carcinoma, $(\mathrm{K})$ uterine corpus endometrial carcinoma, and $(\mathrm{L})$ PFI forest plots for HMMR expression in pan-cancer. 

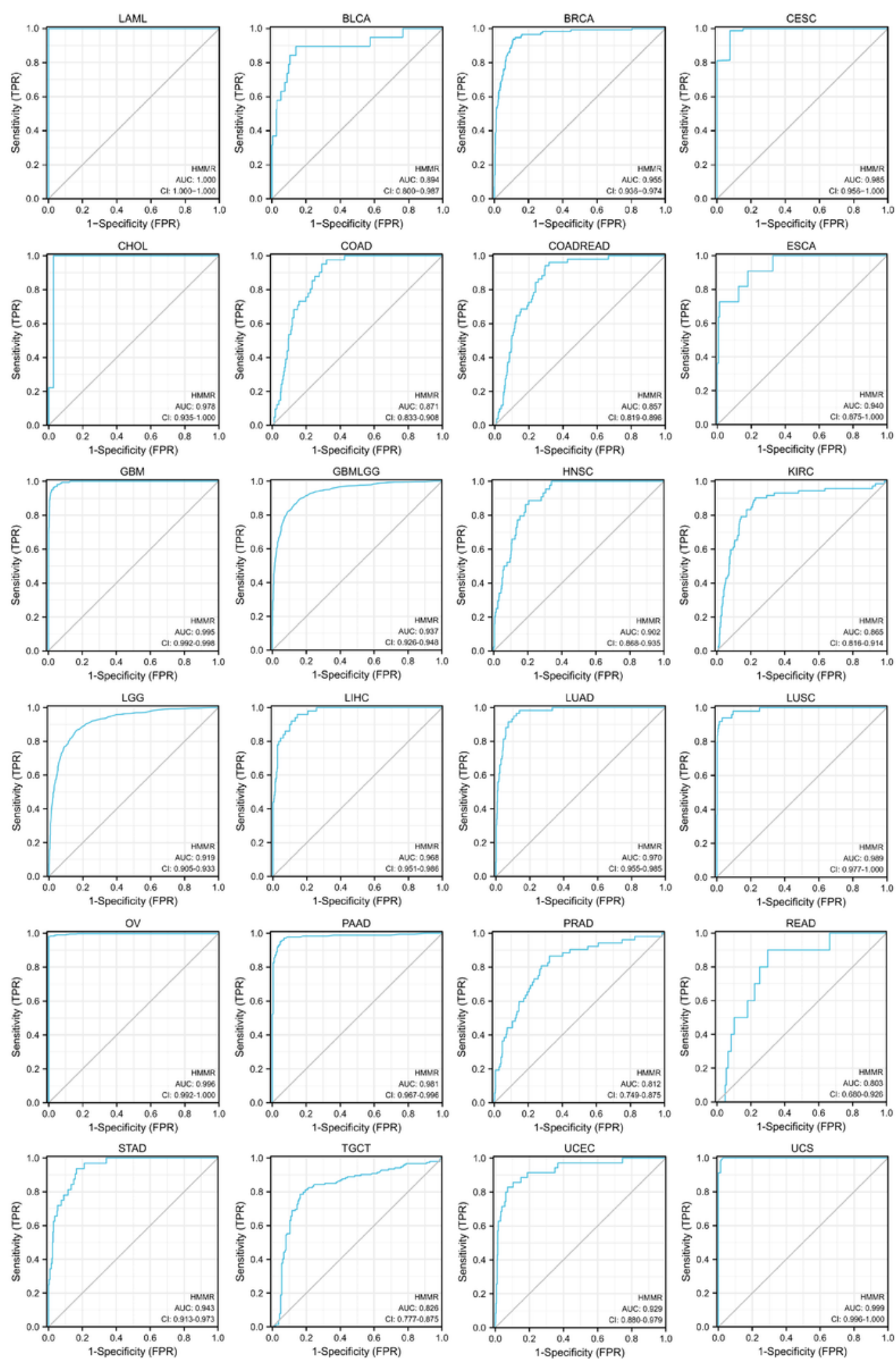

\section{Figure 4}

The ROC curve of HMMR in predicting tumor and normal outcomes. For Areas Under the ROC Curve (AUC) (which are between 0.5 and 1 ) the closer the value is to 1 , the better the diagnostic value. HMMR displays poor accuracy for AUCs between 0.5 and 0.7. HMMR displays certain accuracy when AUC is between 0.7 and 0.9 , and good accuracy when AUC is above 0.9 . 

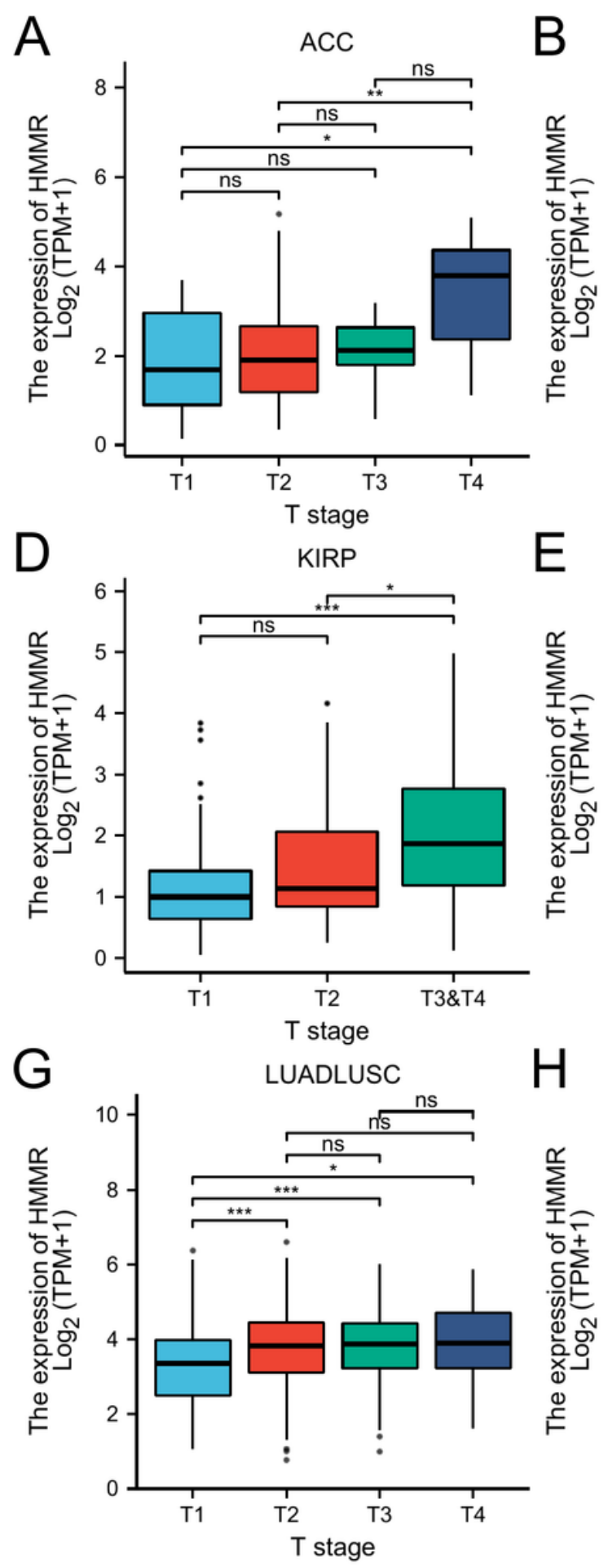

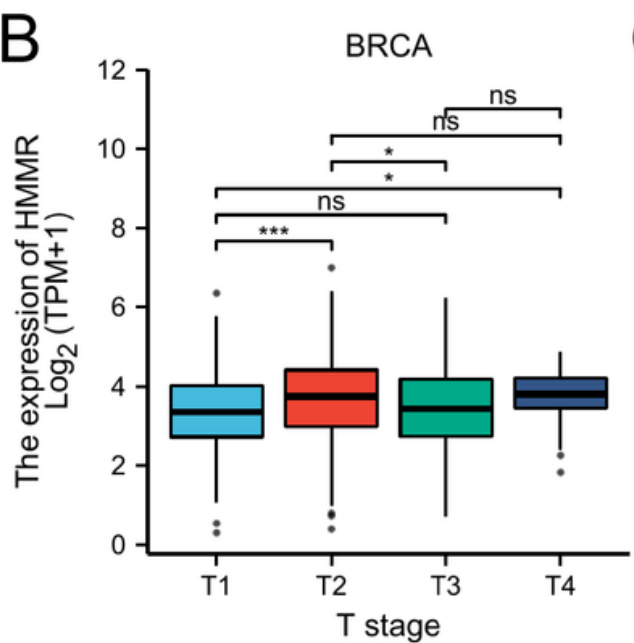

$\mathrm{E}$
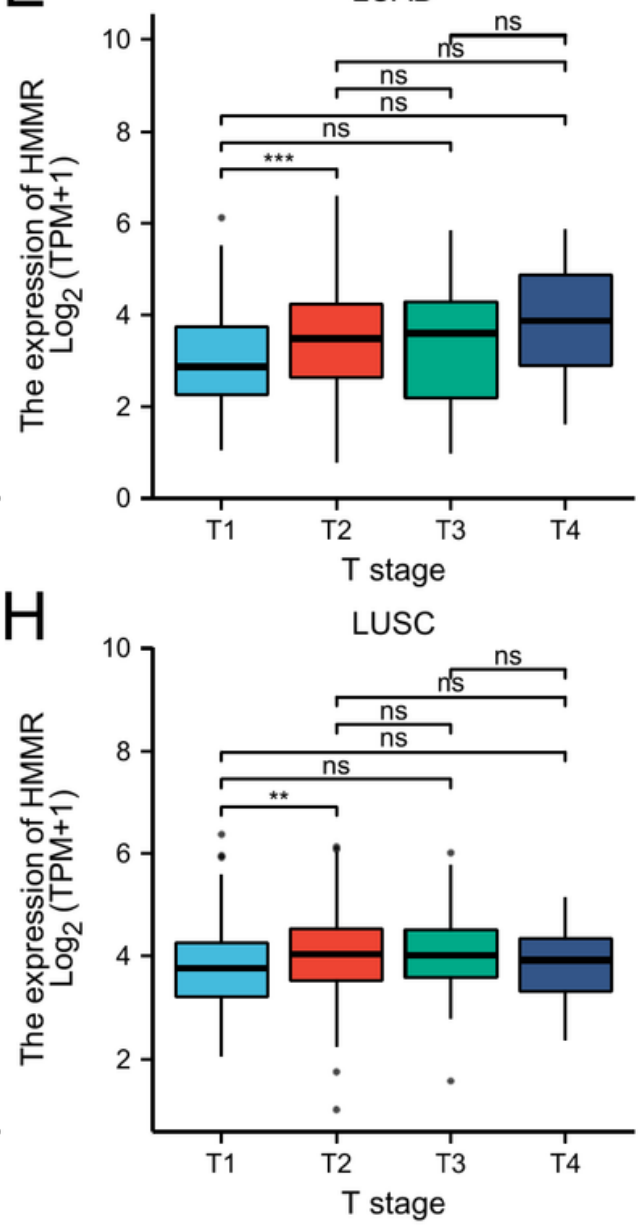

C KIRC

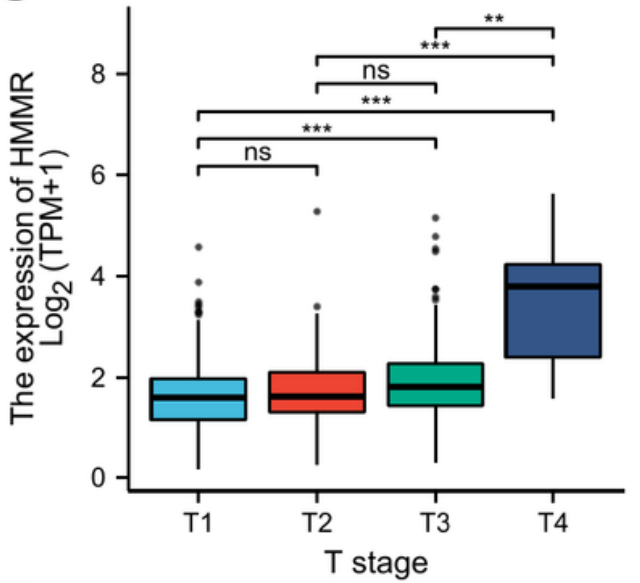

$\mathrm{F}$

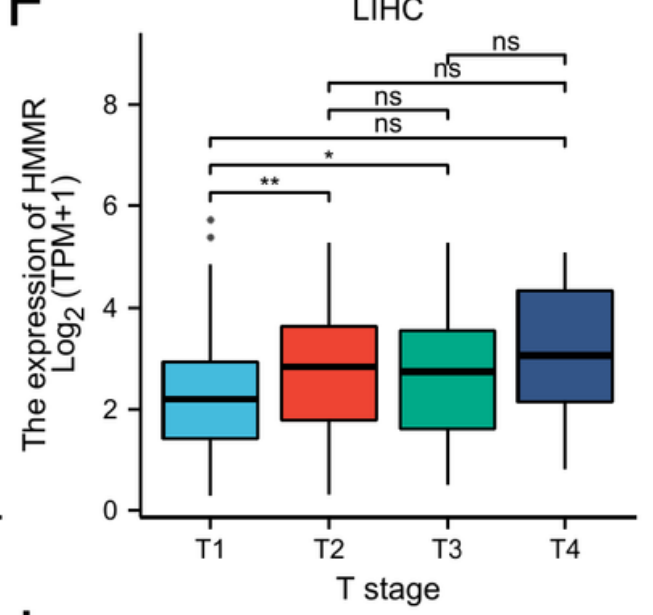

I

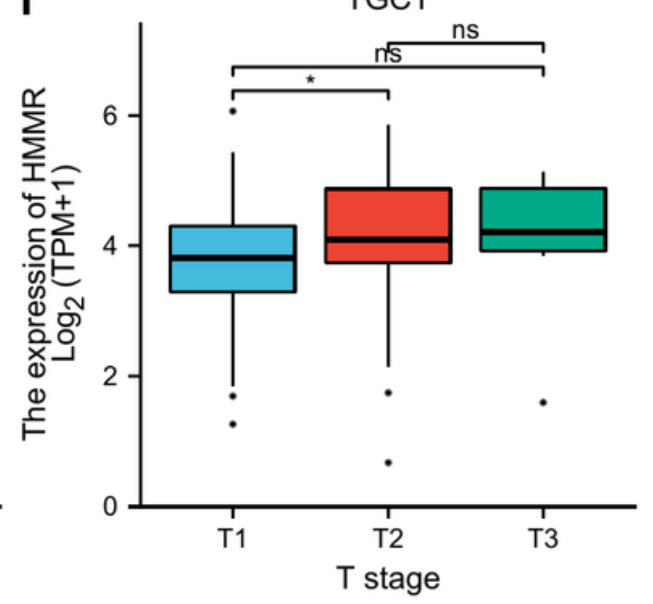

Figure 5

A statistical correlation analysis between HMMR and the pathological T stage. The horizontal axis represents different T stage, the vertical axis represents HMMR expression. $n s, p \geq 0.05 ; *, p<0.05 ; * \star, p<$ $0.01 ; * \star \star, p<0.001$. 
B
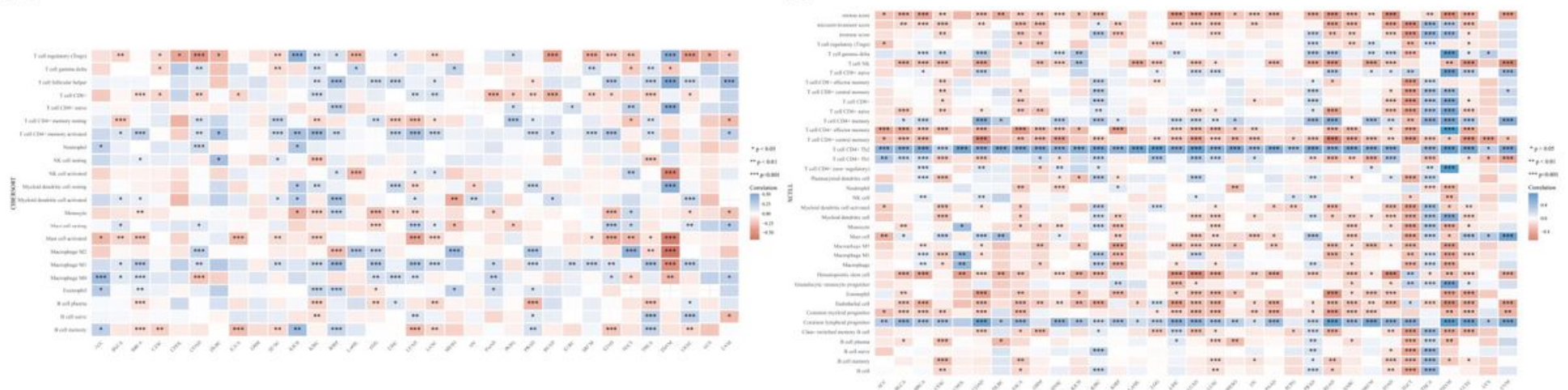

C

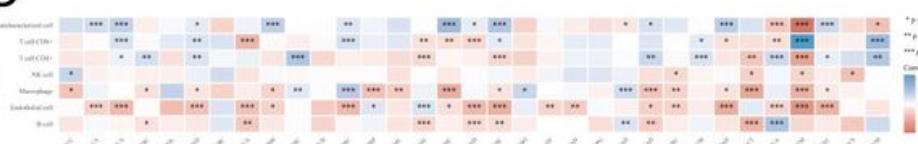

D

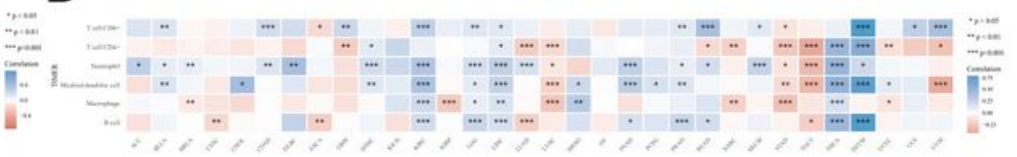

E

$\mathrm{F}$
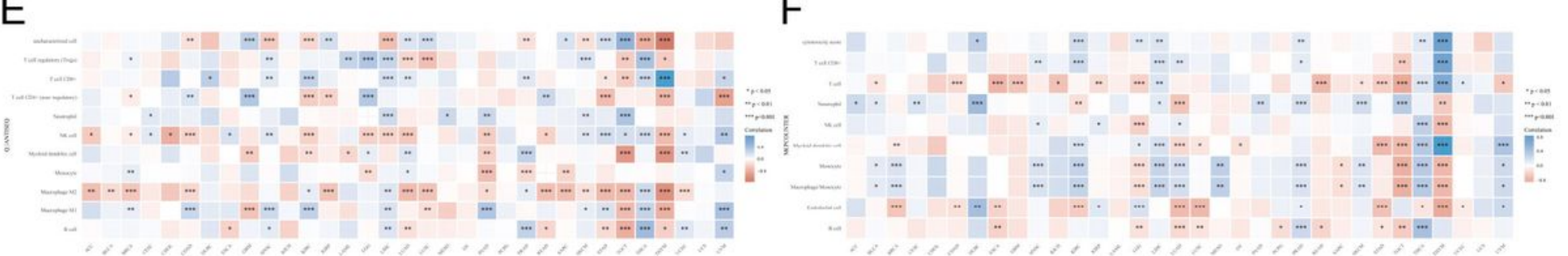

Figure 6

The correlation between HMMR and different immune cells in various tumor types. The horizontal axis represents different tumor types, the vertical axis represents different immune scores, different colors represent the correlation coefficient, negative values indicate an inverse correlation, and positive values indicate a positive correlation. The stronger the correlation, the darker the color. Asterisks represent the degree of importance ( $\left.{ }^{*} p\right)$. ${ }^{*}, p<0.05 ; * \star, p<0.01 ; * \star \star, p<0.001$. (A) CIBERSORT, (B) $x$ Cell, (C) EPIC, (D) TIMER, (E) quanTIseq, and (F) MCP-counter. 


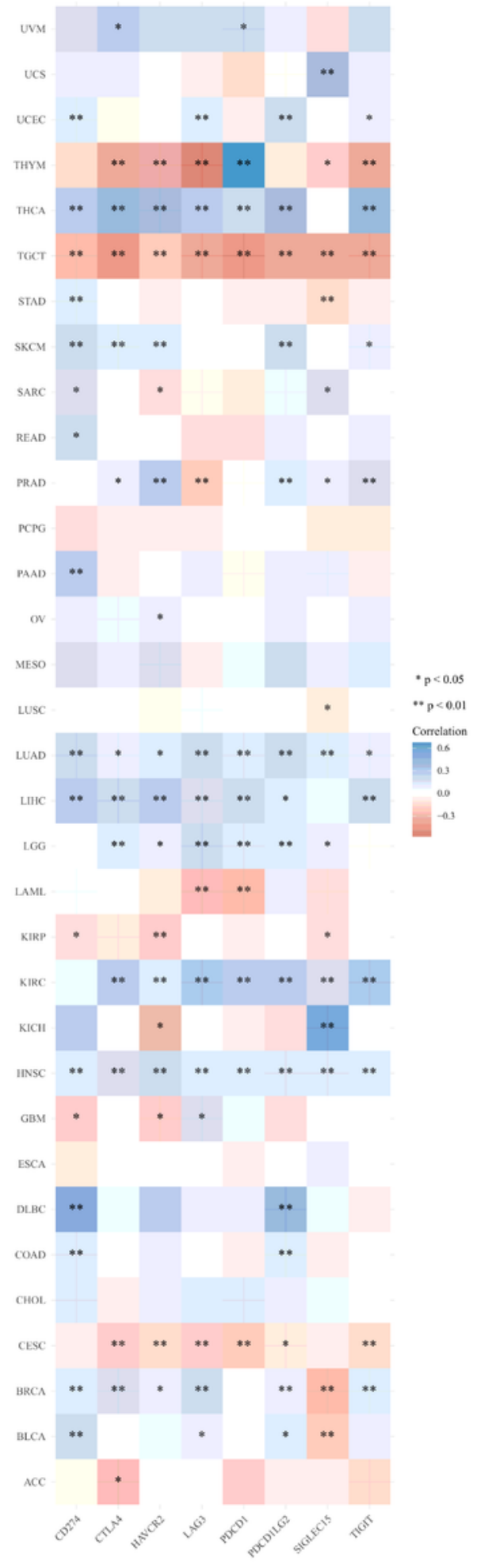

\section{Figure 7}

A heat map for the correlation between HMMR and immune checkpoint related genes in 33 tumor types from TCGA. The horizontal axis provides various immune checkpoint-related genes, and the vertical axis provides various tumor types. Each single box in the figure indicates the correlation between HMMR expression and the expression of immune checkpoint related genes in each tumor. Different colors 
represent different correlation coefficients. The stronger the color, the stronger the correlation. Asterisks represent the level of importance $\left({ }^{*} p\right), *, p<0.05 ; * *, p<0.01$, and ${ }^{* *}, p<0.001$.

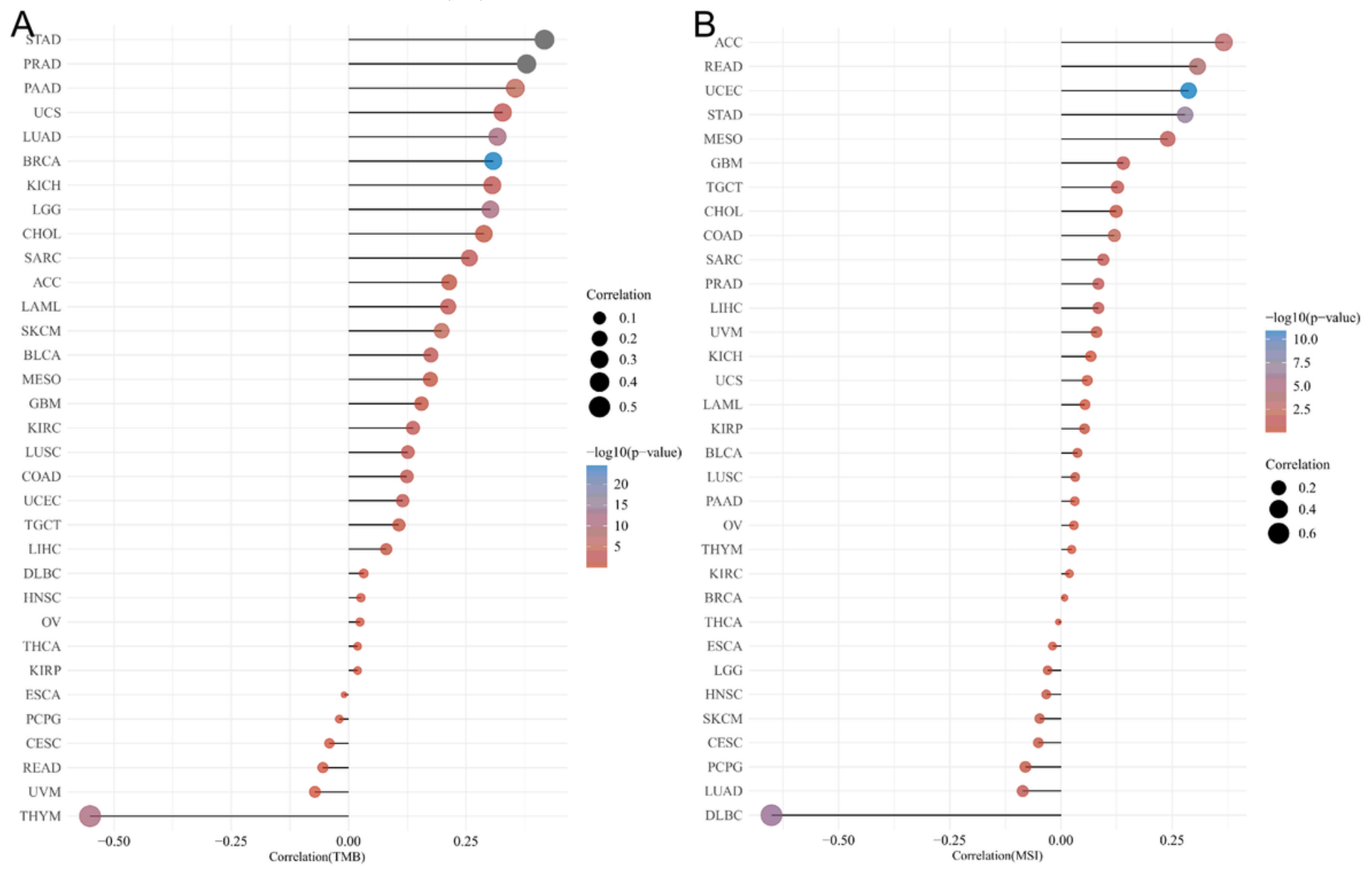

\section{Figure 8}

A correlation analysis for HMMR with TMB (A) and MSI (B) for different tumor types.

A

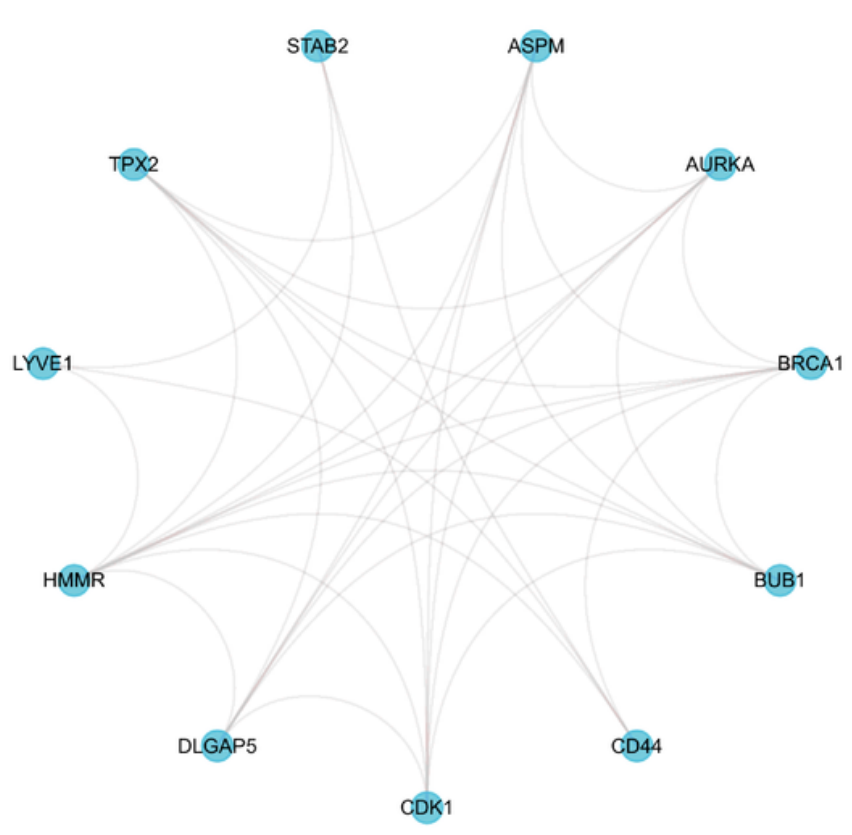

$\mathrm{B}$

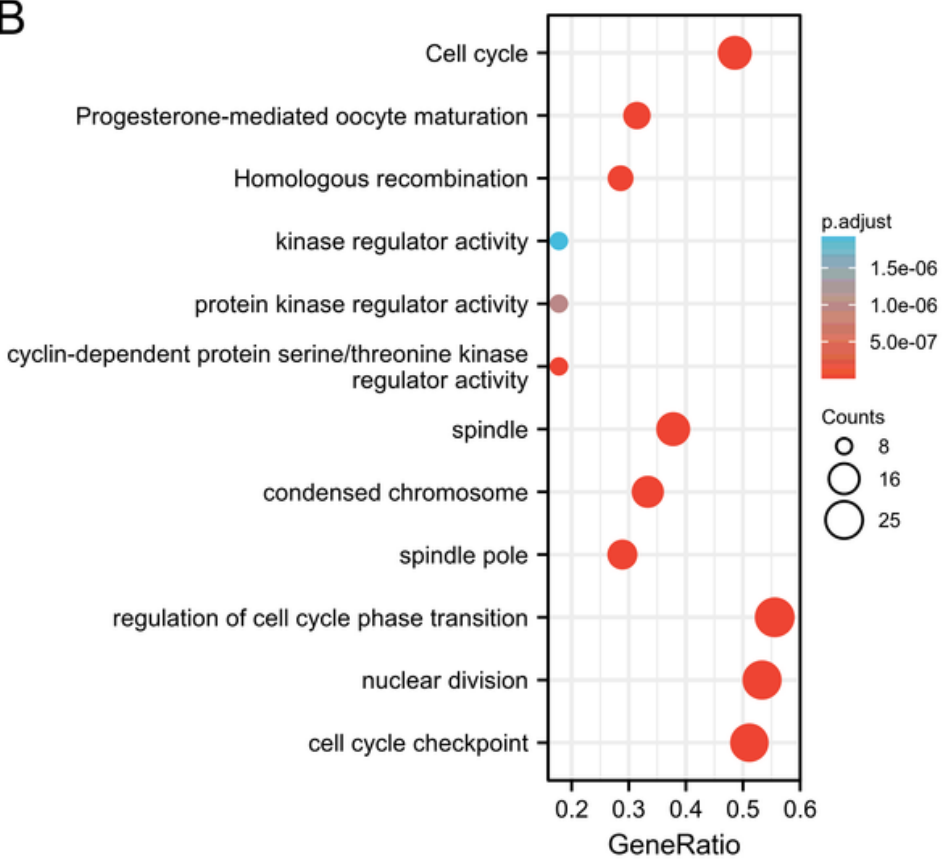




\section{Figure 9}

(A) The PPI network for the top 10 proteins associated with HMMR. (B) A GO/KEGG enrichment analysis for the top 50 proteins associated with HMMR. 